\title{
Capsule Commentary on Herzig et al.: Risk Factors for Nosocomial Gastrointestinal Bleeding and Use of Acid-Suppressive Medication in Non-Critically III Patients
}

\author{
Sarah Nickoloff, MD \\ $J$ Gen Intern Med 28(5):710 \\ DOI: $10.1007 / \mathrm{s} 11606-013-2351-2$ \\ (c) Society of General Internal Medicine 2013
}

Medical College of Wisconsin, Milwaukee, WI, USA.

I $t$ is well established that certain subsets of patients in the intensive care unit should receive prophylactic acidsuppressive medication to decrease risk for nosocomial gastrointestinal bleeding. ${ }^{1}$ However, there is a paucity of literature regarding prophylaxis for nosocomial gastrointestinal bleeding in non-critically ill patients. While recent studies have shown a low incidence of nosocomial bleeding, clinically significant gastrointestinal bleeds remain an important cause of hospital morbidity and mortality, ${ }^{2,3}$ although acid-suppressive medications are not without their own attendant risks.

Herzig $^{4}$ and colleagues retrospectively reviewed 75,723 non-critically ill admissions to a single Boston medical center, collected information on prospectively defined risk factors for nosocomial gastrointestinal bleeding, and developed a scoring system to predict bleeding risk. They experienced $203(0.22 \%)$ nosocomial bleeds. Risk factors included age $>60$, male sex, liver disease, acute renal failure, sepsis, being on a medicine service, prophylactic anticoagulants, and coagulopathy. Each risk factor was given 2 points, and the authors recommended prophylaxis for patients with more than 10 points to prevent nosocomial bleeding (NNT: 95).

While this is a clinically important study, it is only a starting point. First, most patients in the cohort were on acidsuppressive medications, making the numbers in some groups rather small. Second, it was a single medical center, and the results may not be generalizable to other settings. In general, prediction rules tend to perform better in the original population than in subsequent ones. Third, even in the highrisk group, nosocomial bleeding rates were quite low at only
$3.3 \%$. Fourth, this is an observational study, and it is impossible to know how many of these bleeds could have been prevented with acid-suppressive medication.

For clinicians considering acid-suppressive medication in non-critically ill patients, this scoring model uses readily available information and provides guidance in targeting patients who are at highest risk and will benefit most from acid-suppressive medication. It is a good start and could be used to reduce the current high rates of acid-suppressive medication use. Further studies, including validation of the scoring model and randomized trials of acid suppressive medications based on patient risk factors, need to be performed.

Conflict of Interest: The author declares that he/she does not have a conflict of interest.

Corresponding Author: Sarah Nickoloff, MD; Medical College of Wisconsin, 9200 W Wisconsin Ave, Milwaukee, WI 53226, USA (e-mail: sarah.nickoloff@va.gov).

\section{REFERENCES}

1. ASHP Therapeutic Guidelines on Stress Ulcer Prophylaxis. ASHP Commission on Therapeutics and approved by the ASHP Board of Directors on November 14, 1998. Am J Health Syst Pharm. 1999;56 (4):347-79.

2. Herzig SJ, Vaughn BP, Howell MD, Ngo LH, Marcantonio ER. Acidsuppressive medication use and the risk for nosocomial gastrointestinal tract bleeding. Arch Intern Med. 2011;171(11):991-7.

3. Gadeer MA, Richter JE, Brotman DJ. Hospital-acquired gastrointestinal bleeding outside the critical care unit: risk factors, role of acid suppression, and endoscopy findings. J Hosp Med. 2006;1(1):13-20.

4. Herzig SJ, Rothberg MB, Feinbloom DB, Howell MD, Ho KKL, Ngo HL, Marcantonio ER. Risk factors for nosocomial gastrointestinal bleeding and use of acid-suppressive medication in non-critically ill patients. J Gen Intern Med. 2013. doi:10.1007/s11606-012-2296-x. 